\title{
ETHYLENE GLYCOL, HAZARDOUS SUBSTANCE IN THE HOUSEHOLD
}

\author{
Jiří Patočka ${ }^{1,2}$, Zdeněk Hon ${ }^{1}$ \\ University of South Bohemia, České Budějovice, Faculty of Health and Social Studies, Czech Republic: Department of \\ Radiology and Toxicology ${ }^{1}$; Institute of Applied Economical Studies, Department of Technical and Environmental \\ Sciences, Prague, Czech Republic ${ }^{2}$
}

\begin{abstract}
Summary: Ethylene glycol is a colorless, odorless, sweet-tasting but poisonous type of alcohol found in many household products. The major use of ethylene glycol is as an antifreeze in, for example, automobiles, in air conditioning systems, in de-icing fluid for windshields, and else. People sometimes drink ethylene glycol mistakenly or on purpose as a substitute for alcohol. Ethylene glycol is toxic, and its drinking should be considered a medical emergency. The major danger from ethylene glycol is following ingestion. Due to its sweet taste, peoples and occasionally animals will sometimes consume large quantities of it if given access to antifreeze. While ethylene glycol itself has a relatively low degree of toxicity, its metabolites are responsible for extensive cellular damage to various tissues, especially the kidneys. This injury is caused by the metabolites, glycolic and oxalic acid and their respective salts, through crystal formation and possibly other mechanisms. Toxic metabolites of ethylene glycol can damage the brain, liver, kidneys, and lungs. The poisoning causes disturbances in the metabolism pathways, including metabolic acidosis. The disturbances may be severe enough to cause profound shock, organ failure, and death. Ethylene glycol is a common poisoning requiring antidotal treatment.
\end{abstract}

Key words: Ethylene glycol; Antifreez; Health risk; Ingestion; Human poisoning; Toxicity; Glycolic acid; Glycoaldehyde; Calcium oxalate crystals; Acidosis; Fomepizole

\section{Introduction}

Ethylene glycol is most commonly found as the primary ingredient of automobile antifreeze and hydraulic brake fluids. The major use of ethylene glycol as an antifreeze is namely in automobiles, in air conditioning systems and in as de-icing fluid for windshields. It is a widely used chemical that is capable of causing significant injury if ingested. It is not intrinsically toxic but requires metabolism. There are various intermediate metabolic products terminating in oxalic acid. Exposure to large amounts of ethylene glycol can damage the kidneys, nervous system, lungs, and heart (21). This noxa has been found in at least 37 of 1,689 National Priorities List sites identified by the Environmental Protection Agency (4).

In clinical practice, poisoning with ethylene glycol is common but the number of poisonous subjects in various countries are very different and registered insufficiently. Toxicological The Czech Information Centre answers annualy about sixty acute questions about drinking of ethylene glycol (14). In the Czech Republic adult men are the most endangered segment of the population. Thirty-three poisonous victims were obtained between 2000 and 2002. Three patients died, and 11 patients developed acute renal failure (mean maximum serum creatinine level $618 \mu \mathrm{mol} / \mathrm{L}$ ).
Upon discharge, serum creatinine levels were still elevated in 10 patients. Antidote ethanol was given in 30 patients. Renal function completely normalized within 20 months after intoxication (13). Ethylene glycol is also a potential agent for chemical terrorism as an ingredient in explosives. Physico-chemical properties, toxicological profile and clinical symptoms of poisoning is discussed in this article.

\section{Ethylene Glycol Characterization}

Ethylene glycol (1,2-ethanediol, ethane-1,2-diol) (CAS Number 107-21-1) is an alcohol with two hydroxyl groups (a diol). Molecular formula $\mathrm{C}_{2} \mathrm{H}_{4}(\mathrm{OH})_{2}$, molar mass 62.068 $\mathrm{g} / \mathrm{mol}$, density $1.1132 \mathrm{~g} / \mathrm{cm}^{3}$. In its pure form, it is an odorless, colorless, syrupy liquid with a sweet taste and viscosity $16.1 \mathrm{mPa}$ s. It is relatively non-volatile liquid, vapor density 2.14 (air = 1), (m.p. $-12.9^{\circ} \mathrm{C}$, b.p. $122-124^{\circ} \mathrm{C}$ ) and is completely soluble in water in all proportions. Soluble in lower aliphatic alcohols, acetone, glycerol and acetic acid, slightly soluble in ether, practically insoluble in chlorinated hydrocarbons, petroleum ether, benzene and oils. Partition coefficinet $\log \mathrm{K}_{\mathrm{ow}}=-1.36$ (18). Ethylene glycol is inflammable liquid with flash point $127^{\circ} \mathrm{C}$ and autoignition temperature $398{ }^{\circ} \mathrm{C}$, explosive limits 3.2-53\%. R-phrases R22, R36, S-phrases S26, S36, S37, S39, S45, S53. It is manu- 
factured from ethylene, via intermediate ethylene oxide, which reacts with water to produce ethylene glycol.

\section{Toxicokinetics}

Dermal exposure, through activities such as changing antifreeze, is the most likely route of exposure to ethylene glycol, but dermal exposure is not likely to lead to toxic effects. On the other hand, inhalation exposure is due to low volatility of ethylene glycol practically excepted. Only oral exposure, through accidental or intentional ingestion, is likely to lead to such effects, and then only if a sufficient amount is swallowed at one time. Ethylene glycol is rapidly and completely absorbed upon ingestion from the gastrointestinal tract and achieves peak concentration within 30 to 60 minutes after oral ingestion. It has a half-life of 2.5 to 4.5 hours and the half-life may be extended to as long as 17 hours in the presence of therapeutic blood ethanol levels (100-200 mg/dL). Ethylene glycol has a volume of distribution $(0.54-0.8 \mathrm{~L} / \mathrm{kg})$ similar to that of total body water. Ethylene glycol is filtered by the renal glomeruli and is passively reabsorbed. Approximately $20 \%$ of ethylene glycol is is eliminated by the kidneys, but the rate of excretion through this route is slow, with a half-life of 18 to 20 hours (3). However, major part of ethylene glycol is metabolized by the liver ( $80 \%$ ) with a short half-life of 3 to 8 hours and its metabolites are actually dangerous (8).

Ethylene glycol is toxic also for numerous animals. Sometimes there are accidental poisoning of domestic animals. Cats and dogs are relative sensitive, unlike mice, rats or guinea pigs $(18,24)$.

\section{Mechanism of Toxic Action}

Ethylene glycol itself has a relatively low degree of toxicity. Its major toxicities are a result of it being transformed to toxic metabolites. The metabolism of ethylene glycol is a multi-step process taking place primarily in the liver (Fig. 1). Compound is metabolised via alcohol dehydrogenase to glycolaldehyde which is subsequently metabolised to glycolate through interaction with aldehyde dehydrogenase. This metabolite is mainly responsible for the metabolic acidosis in ethylene glycol poisoning. Glycolate is metabolised by various pathways, including one to oxalate which rapidly precipitates with calcium in various tissues and in the urine. Indeed glycolate may be transformed also into the safer glutamate or $\alpha$-ketoadipic acid metabolites. Ethylene glycol toxicity is complex and not fully understood (26) At least four toxic metabolites are result of ethylene glycol biotransformation in human body: glycoaldehyde, glycolic acid, glyoxylic acid, and oxalic acid. These metabolites cause tissue destruction, primarily from calcium oxalate crystal deposition, and metabolic abnormalities, specifically a high anion-gap metabolic acidosis, lactic acidosis, and hypocalcemia (10). Oxalic acid combines with calcium to form calcium oxalate crystals, which deposit in the kidneys. This can result in hypocalcemia, hematuria, proteinuria, and crystalluria, increased creatinine and renal failure.

Calcium oxalate crystal formation is one of the toxic effects of ethylene glycol poisoning. The shape of the urinary crystals can be prismatic and resemble hippurate rather than the expected dipyramidal calcium oxalate dihydrate. X-ray crystallography positively identified them as calcium oxalate monohydrate (6).

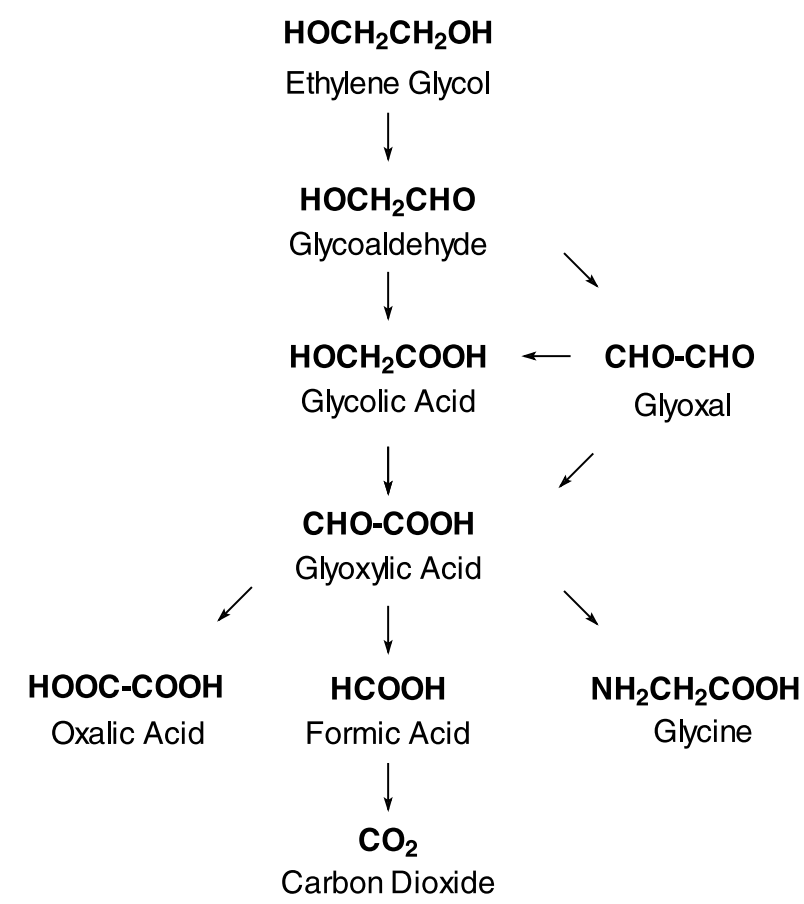

Fig. 1: Major pathway for the metabolism of ethylene glycol in the liver. The first step in this pathway is catalyzed by alcohol dehydrogenases.

\section{Human Poisoning and Clinical Symptoms}

Ethylene glycol is toxic, and its ingestion should be considered a medical emergency (28). Ethylene glycol may be swallowed accidentally, or it may be taken deliberately in a suicide attempt or as a substitute for drinking alcohol (ethanol) (16). Misuse of ethylene glycol as excuse of alcohol drinks is relatively frequent in certain specific communities. For example, in 2005, more than 6,000 exposures to ethylene glycol were reported in the U.S., resulting in $41 \mathrm{fa}-$ talities (15). This document only includes reported cases, therefore the actual number of ethylene glycol exposures may be greater. Drinking ethylene glycol is a medical emergency and poisoning by this substance requires antidotal treatment (2).

The first symptom of ethylene glycol ingestion is similar to the feeling caused by drinking alcohol 11). Within a few hours, more toxic effects become apparent. Symptoms may include nausea, vomiting, convulsions, stupor, or even coma. Ethylene glycol toxicity should be suspected in anyone who 
is severely ill after drinking an unknown substance, especially if they initially appear drunk and do not have a smell of alcohol on their breath.

The major danger from ethylene glycol is following ingestion. In some countries, a bittering agent (denatonium benzoate) or colouring substane (fluorescein), is generally tacked to ethylene glycol preparations as a disgusting compound to prevent accidental ingestion. The lethal dose in adults is $1-1.5 \mathrm{~mL} / \mathrm{kg}(13,27)$.

The clinical course of ethylene glycol poisoning has been described in three stages: an early neurological stage, followed by an intermediate cardiopulmonary stage and finally, a late renal stage. However, in many instances, there may be considerable overlap among the three stages:

Stage 1 (Minutes - 12 hours)

In this early neurological stage CNS toxicity predominates with transient inebriation and euphoria, similar to ethanol intoxication. Nausea and vomiting may also occur. As ethylene glycol metabolism progresses, these symptoms are replaced by metabolic acidosis and CNS depression. About 4-12 hours after ingestion, symptoms associated with the accumulation of the toxic metabolites will begin to predominate. Neurological symptoms may include change in eye movement (nystagmus), loss of coordination (ataxia, paralysis), and in severe cases may include coma associated with hypotonia, hyporeflexia and seizures (1). Neurological symptoms mainly due to aldehyde metabilites. An elevated serum osmolarity is seen early in this phase. Calcium oxalate crystals may be present.

Stage 2 (12-24 hours)

During the second stage of poisoning, tachykardia and mild hypertension commonly occurs. Other effects include anion gap metabolic acidosis with compensatory hyperventilation, and may be accompanied by multiple organ failure. Death most commonly occurs during this stage (1).

Stage 3 (>24 hours)

The symptoms of the third stage are the result of renal injury. This renal phase is characterized by acute tubular necrosis and renal failure. Oliguria, anuria, hematuria, proteinuria, and crystalluria are common. In the event of renal failure, weeks to months of hemodialysis may be nesessary while renal function slowly returns (1).

Freilich et al. (5) described a case study of ethylene glycol intoxication with neurological, radiological, and neuropsychological findings several days to weeks after initial ingestion and again at follow-up six months later. Neuropsychological assessment at three to four weeks post admission indicated global cognitive impairment with particular deficits in attention, processing speed, constructional ability, language, and memory retrieval. Results at six months follow-up indicated partial improvement in overall cognitive functioning with remaining deficits in processing speed and constructional ability.
Studies with people who used ethylene glycol did not show carcinogenic effects. Animal studies also have not shown these chemicals to be carcinogens (24).

\section{Diagnosis of Poisoning}

Early diagnosis of ethylene glycol poisoning is crucial in order to prevent morbidity and mortality (31). Diagnosis of ethylene glycol toxicity is usually made through a combination of blood, urine, and other tests such as blood gas analysis. Ethylene glycol levels are important but reasonable only in some laboratories. If ethylene glycol levels are not available within a few hours, treatment should be initiated until the lab results become available. Ethylene glycol levels $>20 \mathrm{mg} / \mathrm{dL}$ are considered toxic, but levels $<20 \mathrm{mg} / \mathrm{dL}$ may still indicate a toxic amount of etghylene glycol has been ingested if significant time has passed since the ingestion.

An elevated serum osmolality may be present within the first hour of ingestion as this is a result of the presence of ethylene glycol itself, not the toxic metabolites, in the serum. As metabolism decreases the ethylene glycol concentration, the serum osmolality will decrease despite worsening systemic toxicity. A significantly elevated serum osmolality can be indicative of an ethylene glycol ingestion but the absence of an elevated serum osmolarity does not rule out the ingestion of this alcohol (30). Ethylene glycol levels and elevated serum osmolarity as the main indicators of poisoning may not always be valid (25).

Electrolytes may be used to determine the presence of an anion gap acidosis. A normal anion gap is $8-16 \mathrm{mEq} / \mathrm{L}$. The anion gap is mainly due to a decrease in serum bicarbonate levels and usually follows the development of acidosis which typically develops within 12 hours of ingestion. Hypocalcaemia due to formation of calcium oxalate can be massive. Cautious interpretation of lactate assay should be done, since wrong results of lactacidemia could lead to misdiagnostic and delay patient treatment (7).

Renal function tests and urinalysis should be done on symptomatic patients. The presence of calcium oxalate crystals in the urine may be help support the diagnosis of ethylene glycol ingestion. Caution is necessary because the monohydrate form of calcium oxalate crystals are similar to sodium urate crystals.

\section{Treatment}

Any ingestion of ethylene glycol needs to be referred to emergency department. The concentration of the product is important in determining potential toxicity. If the concentration is $>20 \%$ then a mouthful could be toxic, and in children anything more than a witnessed taste or lick may be a cause for concern. Decontamination is generally of little value since ethylene glycol is so rapidly absorbed from the GI tract. However lavage may be beneficial if performed within 60 minutes of a large ingestion. Prognosis is excel- 
lent in both poisonings provided that there is early treatment with alkali to combat acidosis, ethanol as an antimetabolite, and haemodialysis to remove the alcohols and their toxic metabolites.

Correction of metabolic acidosis. Acidosis may be corrected by use of sodium bicarbonate (19). The patient who arrived at the hospital six hours after suicide ingestion of $500 \mathrm{ml}$ of ethylene glycol (serum EG level $0.282 \mathrm{~g} / \mathrm{l}$ ) with metabolic acidosis $(\mathrm{pH}=7.176)$ and signs of nephrotoxicity (serum creatinine $168 \mu \mathrm{mol} / 1$ ) has been fully recovered (12).

Ethylene glycol oxidation blocking. Oxidation of ethylene glycol by alcohol dehydrogenase is a critical step in its conversion to oxalate, which produces metabolic acidosis and ultimately renal failure due to the deposition of oxalate in the renal tubules. Therefore, if the oxidation of ethylene glycol to oxalate can be blocked, the fatal toxicity due to the ingestion of this compound can be prevented. Detailed studies of the enzymatic oxidation of ethylene glycol demonstrate that ethyl alcohol, the natural substrate, is a potent inhibitor of the oxidation of ethylene glycol. Hence, low concentrations of ethyl alcohol prevent the oxidation of large concentrations of ethylene glycol (32).

Ethanol acts as a competitive inhibitor to the active site of the alcohol dehydrogenase that converts ethylene glycol to its toxic metabolites. Once ethanol binds, the ethylene glycol is harmlessly excreted out of the body but the serum ethanol level must be monitored frequently. Therapeutic ethanol is administered in a bolus followed by a continuous infusion. Initially, 7.5 to $10 \mathrm{~mL} / \mathrm{Kg}$ of $10 \%$ ethanol is administered over 30 minutes. Then, a continuous infusion of 1 to $2 \mathrm{~mL} / \mathrm{Kg} / \mathrm{hr}$ of $10 \%$ ethanol is infused until the patient has eliminated all of the ethylene glycol from his serum. It is important to keep the serum ethanol level at 100 to 150 $\mathrm{mg} / \mathrm{dL}$ so as to completely inhibit the alcohol dehydrogenase enzyme (2). Peroral application of ethanol is recommended in the first aid: Initially $1.5 \mathrm{ml} / \mathrm{Kg}$ of $50 \%$ ethanol in a bolus and then maintenance dose $0.2-0.3 \mathrm{ml} / \mathrm{Kg} / \mathrm{hr}$.

Fomepizole (Antizole) also blocks alcohol dehydrogenase and is considered as the drug of choice for antidotal therapy for ethylene glycol toxicity. Fomepizole is administered oraly in dose $15 \mathrm{mg} / \mathrm{Kg}$ (up to 1 gramme) initially, then $10 \mathrm{mg} / \mathrm{Kg}$ every $12 \mathrm{~h}$ in four doses, and then $15 \mathrm{mg} / \mathrm{Kg}$ every 12 hours until ethylene glycol level $<10 \mathrm{mg} / \mathrm{dL}$ (23, 30).

Extracorporeal removal of the toxic substances. In patients who are symptomatic, hemodialysis or hemoperfusion should be considered. Hemodialysis efficiently clears ethylene glycol and toxic byproducts and corrects acidosis. Use of sodium bicarbonate enhances elimination of toxic byproduct by correction of acidosis (9). The i.v. administration of ethanol, combined with hemodialysis, appears to be effective and safe for the management of patients with ethylene glycol poisoning (20).

Supportive therapy. Pyridoxine in dose $50 \mathrm{mg}$ every 6 hours may inhibit metabolism of glycolic acid to oxalic acid by acting as cofactor in metabolism of glycolic acid to nonoxalate byproducts. Thiamine in dose $100 \mathrm{mg}$ every 6 hours is recommended to stimulate the conversion of glyoxylate to alpha-hydroxy-beta-ketoadipate, a non-toxic metabolite (17). Activated charcoal is ineffective and is nonrecommended because ethylene glycol is not likely adsorbed by this agent (22).

\section{Conclusions}

Ethylene glycol is a synthetic liquid substance used to make antifreeze and de-icing solution. Ethylene glycol is the major ingredient of almost all radiator fluid products in the United States. It is odorless, but has a sweet taste, which is why some animals are attracted to it. Substance is a relatively nontoxic compound before it is metabolized. The metabolites cause the distinctive toxicity associated with this compound. Ethylene glycol is metabolized by alcohol dehydrogenase to form glycoaldehyde. Through interaction with aldehyde dehydrogenase, ethylene glycol is then metabolized to glycolic acid. A profound acidosis often ensues and is attributable to the glycolic acid in circulation. This glycolate is then transformed into glyoxylic acid and this molecule may be transformed into the highly toxic oxalate.

A review of the literature for ethylene glycol indicated that the stages of oral ethylene glycol poisoning in humans are well understood and documented. There is adequate knowledge of ethylene glycol metabolism to permit successful treatment of ethylene glycol intoxication. The majority of the studies in humans represent descriptions of case studies of accidental or intentional poisoning. Animal studies corroborate human findings and were used to provide quantitative data to support observations made in humans.

\section{References}

1. Barceloux DG, Krenzelok EP, Olson K, Watson W. American Academy of Clinical Toxicology Practice guidelines on the treatment of ethylene glycol poisoning. J Toxicol Clin Toxicol 1999;37:537-60.

2. Brent J. Current management of ethylene glycol poisoning. Drugs 2001;61: 979-88.

3. Eder AF, McGrath CM, Dowdy YG et al. Ethylene glycol poisoning: Toxicokinetic and analytical factors affecting laboratory diagnosis. Clin Chem 1998;44:168-77.

4. EPA: CERCLA Priority list of hazardous substances http://www.atsdr.cdc.gov/ cercla/.

5. Freilich BM, Altun Z, Ramesar C, Medalia A. Neuropsychological sequelae of ethylene glycol intoxication: a case study. Appl Neuropsychol 2007;14:56-61.

6. Godolphin W, Meagher EP, Sanders HD, Frohlich J. Unusual calcium oxalate crystals in ethylene glycol poisoning. Clin Toxicol. 1980 Jun;16(4):479-86.

7. Graine H, Toumi K, Roullier V, Capeau J, Lefevre G. Interference of ethylene glycol on lactate assays [Article in French] Ann Biol Clin (Paris) 2007;65:421-4.

8. Hess R, Bartels MJ, Pottenger LH. Ethylene glycol: an estimate of tolerable levels of exposure based on a review of animal and human data. Arch Toxicol 2004; 78:671-80.

9. Holubek WJ, Hoffman RS, Goldfarb DS, Nelson LS. Use of hemodialysis and hemoperfusion in poisoned patients. Kidney Int. 2008; 74: 1327-34.

10. Ishihara K, Szerlip HM. Anion gap acidosis. Semin Nephrol 1998;18:83-97.

11. Jacobsen D, McMartin KE. Mechanism of toxicity, clinical course, diagnosis and treatment. Med Toxicol 1986;1:309-34.

12. Křenová M, Pelclová, D. Complete recovery after repeated suicidal ethylene glycol ingestion. Prague Med Rep 2006;107:130-6.

13. Křenová M, Pelclová D, Navrátil T, Merta M, Tesař V. Ethylene glycol poisoning in the Czech Republic (2000-2002). Blood Purif 2006;24:180-184. 
14. Křenová M, Pelclová D. Severe intoxications with ethylene glycol and chlorinated aliphatic hydrocarbons [Article in Czech] Cs Prac Lek 2005:4:198-202.

15. Lai MW, Klein-Schwartz W, Rodgers GC et al. 2005 Annual Report of the American Association of Poison Control Centers' National Poisoning and Exposure Database. Clin Toxicol 2006;44:803-932.

16. Leth PM, Gregersen M. Ethylene glycol poisoning. Forensic Sci Int. 2005; 155:179-84.

17. Lheureux P, Penaloza A, Gris M. Pyridoxine in clinical toxicology: a review. Eu J Emerg Med 2005;12:78-85.

18. Lide DR. CRC handbook of chemistry and physics. $86^{\text {th }}$ ed. Boca Raton, FL: Taylor \& Francis Group, 2005. 3-232.

19. Lim S. Metabolic acidosis. Acta Med Indones 2007;39:145-50.

20. Lister D, Tiemey M, Dickinson G. Effectivenes of iv ethanol therapy combined with hemodialysis in the treatment of methanol and ethylene glycol poisoning. Canad J Hosp Pharmac 2005; 58: 126-9.

21. Lovrić M, Granić P, Cubrilo-Turek M, Lalić Z, Sertić J. Ethylene glycol poisoning. Forensic Sci Int 200;170:213-5.

22. McMahon DM. Toxic alcohol ingestions: Focus on ethylene glycol and methanol. Adv Emerg Nursing J 2009; 31: 206-13.

23. Mégarbane B, Houzé P, Baud FJ. Oral fomepizole administration to treat ethylene glycol and methanol poisonings: Advantages and limitations. Clin Toxico 2008;46:1097.
24. National Toxicology Program. NTP Toxicology and Carcinogenesis Studies of Ethylene Glycol (CAS No. 107-21-1) in B6C3F1 Mice (Feed Studies). Nat Toxicol Program Tech Rep Ser 1993;413:1-177.

25. Ostapenko IuN, Litvinov NN, Rozhkov PG, Il'iashenko KK, Gol'dfarb IuS. Problems in the standardization of inpatient health care in clinical toxicology [Article in Russian]. Anesteziol Reanimatol. 2008; 6: 11-5.

26. Poldelski V, Johnson A, Wright S, Rosa VD, Zager RA. Ethylene glycol-mediated tubular injury: identification of critical metabolites and injury pathways. Am J Kidney Dis 2001;38:339-48

27. Sheftel VO. Indirect food additives and polymers. Migration and Toxicology. Lewis Publishers, Boca Raton, FL. 2000, p. 726

28. Ting SMS, Ching I, Nair H, Langman G, Suresh V, Temple RM. Early and late presentations of ethylene glycol poisoning. Am J Kidney Dis 2009;53 1091-7.

29. Velez LI, Gracia R, Neerman MF. Ethylene glycol poisoning: current diagnostic and management issues. J Emerg Nur. 2007;33:342-5.

30. Velez LI, Shepherd G, Lee YC, Keyes DC. Ethylene glycol ingestion treated only with fomepizole. J Med Toxicol 2007:3:125-8.

31. Verelst S, Vermeersch P, Desmet K. Ethylene glycol poisoning presenting with a falsely elevated lactate level. Clin Toxicol (Philadelphia, Pa.) 2009;47:236-8.

32. Wacker WEC, Haynes H, Druyan R, Fisher W, Coleman JE. Treatment of ethylene glycol poisoning with ethyl alcohol. JAMA 1965; 194: 1231-3.

Received: $19 / 08 / 2009$

Accepted in revised form: 10/02/2010.

\section{Corresponding author:}

Prof. RNDr. Jiří Patočka, DrSc., Jílovice 31, 51772 Jílovice u Dobrušky, Czech Republic; e-mail: prof.patocka@gmail.com 